\title{
CURVATURE AND FINITE DOMINATION
}

\author{
MICHAEL WEISS \\ (Communicated by Christopher Croke)
}

\begin{abstract}
Upper bounds obtained by Gromov on the Betti numbers of certain closed Riemannian manifolds are shown to be upper bounds on the minimum number of cells in $C W$-spaces dominating such manifolds.
\end{abstract}

In [Gro1], Gromov obtains a bound on the sum of the Betti numbers of a closed Riemannian manifold $V$ in terms of a lower bound on the sectional curvature and an upper bound on the diameter. In more detail: Fix a field $F$, and let $\beta_{i}=\beta_{i}(V ; F)$ be the dimension over $F$ of $H_{i}(V ; F)$. Let $D=D(V)$ be the diameter of $V$.

Theorem A [Gro1]. There exists a constant $\mathcal{C}=\mathcal{C}(n)$ such that every closed connected Riemannian n-manifold $V$ satisfies

$$
\sum_{0}^{n} \beta_{i} \leq \mathcal{C}^{1+\kappa D}
$$

provided the sectional curvature of $V$ is bounded from below by $-\kappa^{2}$, where $\kappa \geq 0$.

Corollary. If $V$ has non-negative sectional curvature, then the sum of the Betti numbers is $\leq \mathcal{C}$.

A stronger theorem can be obtained with a small change in Gromov's proof. Terminology: For spaces $X$ and $Y$, we say that $X$ dominates $Y$ if there exist maps

$$
Y \stackrel{i}{\longrightarrow} X \stackrel{r}{\longrightarrow} Y
$$

such that $r i$ is homotopic to the identity.

Theorem B. There exists a constant $\mathcal{C}=\mathcal{C}(n)$ such that every closed connected Riemannian $n$-manifold $V$ can be dominated by a $C W$-space $X$ having at most

$$
\mathcal{C}^{1+\kappa D}
$$

cells. (Assume as before that the sectional curvature of $V$ is bounded from below by $-\kappa^{2}$, where $\kappa>0$.)

Note that Theorem B implies an upper bound for the minimum number of generators of $\pi_{1}(V)$. This is in agreement with [Gro2], but less explicit.

Received by the editors February 1, 1994 and, in revised form, February 22, 1994 and August 9, 1994 .

1991 Mathematics Subject Classification. Primary 53C21, 53C20; Secondary 57Q10.

Key words and phrases. Positive curvature, Betti numbers, homotopy direct limits. 
The referee has asked me to point out that Theorem $\mathrm{B}$ explains better than Theorem A how a lower bound on the sectional curvatures of $V$ restricts the topological complexity of $V$. It gives an upper bound on the minimum number of cells in a $C W$-space $X$ dominating $V$, but there is no bound for the complexity of the attaching maps for the cells of $X$. For example, in dimension 3 there are infinitely many different homology types of compact Riemannian manifolds of constant sectional curvature 1 (lens spaces). In dimension 7 , there are infinitely many different homology types of compact simply connected Riemannian manifolds having strictly positive sectional curvature (the examples of Allof and Wallach [AlWa]).

The referee has also drawn my attention to [Abr1] and [Abr2]. Abresch extended Gromov's result to asymptotically non-negatively curved manifolds (which are complete by definition, but not always closed). He obtained more explicit bounds on the Betti numbers. His result is

$$
\sum_{i} \beta_{i}\left(V^{n}\right) \leq c(n) \cdot \exp \left(\frac{15 n-13}{4} \cdot b_{1}\left(V^{n}\right)\right)
$$

where $b_{1}\left(V^{n}\right)$ is a real number (not a Betti number) measuring to some extent the "amount" of negative curvature in $V$ and, in Abresch's own words,

$$
\begin{aligned}
& \text { the function } c(n) \text { can be effectively estimated by } \\
& \text { an expression which grows exponentially in } n^{3} \text {. }
\end{aligned}
$$

If $V$ is closed, with diameter $D$ and sectional curvature bounded from below by $-\kappa^{2}$ everywhere, then $b_{1}(V) \leq \kappa D$ by $[\mathrm{Abr} 1,2.3]$.

Again, a small change in Abresch's proof shows that inequality (*) and the estimate for $c(n)$ remain correct if the sum of the Betti numbers is replaced by the minimum number of cells in a $C W$-space dominating $V$.

The changes should be made in $\S 2.3$ of [Gro1], and in $\S 1$ of [Abr2]. This is where the Leray spectral sequence appears. Gromov refers to [Groth] for details. Grothendieck's account is of course "homological". A more geometric explanation of the Leray spectral sequence (using homotopy direct limits) is available. This is where we start.

\section{The LERAY SPECTRAL SEQUENCE}

Let $\mathcal{A}$ be a simplicial complex. We shall regard $\mathcal{A}$ as a category: objects are the simplices of $\mathcal{A}$, and morphisms are the inclusion maps. For a contravariant functor $\mathcal{Z}$ from $\mathcal{A}$ to the category of spaces, let

$$
|\mathcal{Z}|:=\left(\coprod_{s \subset \mathcal{A}} \mathcal{Z}(s) \times s\right) / \sim
$$

where the coproduct runs over all simplices $s \subset \mathcal{A}$ and $\sim$ stands for the "usual" relations, $\left(f^{*} a, b\right) \sim(a, b)$ whenever $a \in \mathcal{Z}\left(s^{\prime}\right), b \in s$, and $f: s \hookrightarrow s^{\prime}$. Note that $|\mathcal{Z}|$ projects to $\mathcal{A}$ by $(a, b) \mapsto b$. The inverse image of the $k$-skeleton of $\mathcal{A}$ under this map is the vertical $k$-skeleton of $|\mathcal{Z}|$, denoted by $|\mathcal{Z}|(k)$.

The construction $|\mathcal{Z}|$ is a special case of a homotopy direct limit. The notion goes back to [Se] and the standard reference is [BK].

With $\mathcal{A}$ and $\mathcal{Z}$ as above, let $X$ be a space and let $\tau: \mathcal{Z} \longrightarrow X$ be a natural transformation (where we think of $X$ as a constant functor on $\mathcal{A}$ ). This induces a map

$$
\tau_{*}:|\mathcal{Z}| \longrightarrow X ; \quad(a, b) \mapsto \tau(a) .
$$


Example 1.2. Let $X$ be a $C W$ - space, and let $\left\{X_{\gamma} \mid \gamma \in \Gamma\right\}$ be a collection of $C W$-subspaces of $X$. Let $\mathcal{A}$ be the nerve of the collection of subspaces: i.e., $\mathcal{A}$ has one $k$-simplex for each finite subset $\Gamma^{\prime} \subset \Gamma$ such that

$$
\bigcap_{\gamma \in \Gamma^{\prime}} X_{\gamma} \neq \emptyset .
$$

Of course, we let $\mathcal{Z}(s)=\bigcap X_{\gamma}$ where the intersection is taken over all $\gamma$ which are vertices of $s$. The inclusions $\mathcal{Z}(s) \subset X$ define a natural transformation

$$
\tau: \mathcal{Z} \longrightarrow X
$$

If $X$ is the union of the $X_{\gamma}$, then $\tau_{*}:|\mathcal{Z}| \longrightarrow X$ is a homotopy equivalence.

The proof consists essentially in showing that the fibers of $\tau_{*}$ are contractible spaces. For $x \in X$, the fiber of $\tau_{*}$ over $x$ is homeomorphic to the full simplex spanned by vertices $\gamma$ such that $x \in X_{\gamma}$.

Example 1.3. Let $X$ be a smooth $n$-manifold, and let $\left\{X_{\gamma} \mid \gamma \in \Gamma\right\}$ be a collection of open subsets of $X$. Define $\mathcal{A}, \mathcal{Z}$ and $\tau$ as before. If $X$ is the union of the $X_{\gamma}$, then $\tau_{*}:|\mathcal{Z}| \longrightarrow X$ is a homotopy equivalence.

The proof is by reduction to the previous example (use triangulations of $X$ ). Details are left to the reader. The smoothness assumption is unnecessary, but it makes the proof easier.

In the situation of 1.2 or 1.3 , assuming that $X$ is the union of the $X_{\gamma}$, we have the canonical filtration of $|\mathcal{Z}|$ by vertical skeletons $|\mathcal{Z}|(k)$. Now a filtration of a space always gives rise to a filtration of its singular chain complex, and then to a spectral sequence converging to the homology of the space. Here we obtain a spectral sequence converging to the homology of $|\mathcal{Z}|$, which is the homology of $X$. This is the Leray spectral sequence. We are not going to use it. We will use the filtration of $|\mathcal{Z}|$ by vertical skeletons.

\section{Cell content}

The reader should now have [Gro1] before his/her eyes, more specifically, $§ 2.3$ of [Gro1]. First a remark on terminology: As I understand it, Gromov means by a ball in the Riemannian manifold $V$ a certain open subset $B=B(x, R)$ of $V$, equipped with the (sometimes additional) structure of a center $x$ and radius $R$. For example, if $V$ is closed and $D$ is the diameter of $V$, then $B(x, 2 D)$ and $B(x, 10 D)$ must be regarded as different balls in $V$, although the underlying subsets of $V$ are both equal to $V$. If $B=B(x, R)$ is a ball in $V$, and $\lambda$ is a positive real number, then $\lambda B$ denotes the ball $B=B(x, \lambda R)$.

In $\S 2.3$, Gromov defines the content of a ball $B$ in $V$ as the rank of the inclusion homomorphism

$$
H_{*}\left(\frac{1}{5} B ; F\right) \longrightarrow H_{*}(B ; F)
$$

Further, he writes:

Quotation 2.1. "Take a ball $B$ and cover the concentric ball $\frac{1}{5} B$ by some open balls $B_{i}$, where $i=1, \ldots, N$, all of the same radius. Consider also the concentric coverings $\left\{\lambda_{j} B_{i}\right\}$, where $j=0,1, \ldots, n+1$ and $\lambda_{j}=10^{j}$. Suppose that all balls 
$5 \lambda_{j} B_{i}$ (where $j=0, \ldots, n+1$ and $i=1, \ldots, N$ ) are contained in $B$, and let the contents of these balls be bounded by a constant $p$, that is

$$
\operatorname{Cont}\left(5 \lambda_{j} B_{i}\right) \leq p
$$

Denote by $J$ the index $\left[\right.$ Gro1,2.2] of the system $\left\{5 \lambda_{n+1} B_{i}\right\}$, where $i=1, \ldots, N$.

The content of $B$ satisfies the following inequality:

$$
\operatorname{Cont}(B) \leq(n+1) p J . "
$$

Definition 2.2. The cell content of a ball $B$ in $V$ is $\leq q$ if there exists a $C W$-space $Y$ with at most $q$ cells, and maps

$$
\frac{1}{5} B \stackrel{f}{\longrightarrow} Y \stackrel{g}{\longrightarrow} B
$$

such that $g f$ is homotopic to the inclusion.

Lemma 2.3. Keeping the hypotheses of 2.1 in all other respects, suppose that the cell contents of the balls $5 \lambda_{j} B_{i}$ (where $j=0, \ldots, n$ and $i=1, \ldots, N$ ) are bounded by a constant $p$. Then the cell content of $B$ is not greater than $p J$.

(This will be proved in the next section.) Now return to the assumptions and notation of Theorem B above; in particular, let $D$ be the diameter of $V$. Lemma 2.3 implies, by arguments identical with Gromov's, that for any $x \in V$ the cell content of $B(x, 10 D)$ is bounded by $\mathcal{C}^{1+\kappa D}$ for suitable $\mathcal{C}$ independent of $V$ (but depending on the dimension $n$ ). Since

$$
B(x, 10 D)=\frac{1}{5} B(x, 10 D)=V \quad \text { "as sets", }
$$

this means that $V$ can be dominated by a cell complex with at most $\mathcal{C}^{1+\kappa D}$ cells.

\section{Proof of THE LEMMA}

Using Example 1.3, we can deduce Lemma 2.3 from the following statement.

Proposition 3.1. Let $\mathcal{A}$ be a compact simplicial complex with $J$ simplices. Let

$$
\mathcal{Z}_{0} \stackrel{T_{0}}{\longrightarrow} \mathcal{Z}_{1} \stackrel{T_{1}}{\longrightarrow} \mathcal{Z}_{2} \rightarrow \cdots \rightarrow \mathcal{Z}_{n} \stackrel{T_{n}}{\longrightarrow} \mathcal{Z}_{n+1}
$$

be a diagram of functors (contravariant, from $\mathcal{A}$ to spaces) and natural transformations. Assume that, for each simplex $s \subset \mathcal{A}$ and each $j \in\{0,1, \ldots, n\}$, the map from $\mathcal{Z}_{j}(s)$ to $\mathcal{Z}_{j+1}(s)$ given by $T_{j}$ has a (strict) factorization

$$
\mathcal{Z}_{j}(s) \stackrel{\alpha_{j, s}}{\longrightarrow} Y_{j, s} \stackrel{\beta_{j, s}}{\longrightarrow} \mathcal{Z}_{j+1}(s)
$$

where $Y_{j, s}$ is homotopy equivalent to a $C W$-space with not more than $p$ cells. Then the map from $\left|\mathcal{Z}_{0}\right|(n)$ to $\left|\mathcal{Z}_{n+1}\right|$ induced by $T_{n} T_{n-1} \cdots T_{1} T_{0}$ has a factorization

$$
\left|\mathcal{Z}_{0}\right|(n) \longrightarrow W \longrightarrow\left|\mathcal{Z}_{n+1}\right|
$$

where $W$ is homotopy equivalent to a $C W$-space with not more than $p J$ cells. 
Interpretation 3.2. Let $\mathcal{A}$ be the nerve of the collection of open sets $\left\{\lambda_{n+1} B_{i}\right\}$ (notation of 2.1 and 2.3 above). Define $\mathcal{Z}_{j}$ by

$$
\mathcal{Z}_{j}(s)=\bigcap_{i \text { vertex of } s} \lambda_{j} B_{i} \quad \text { for } s \subset \mathcal{A} \text { and } 0 \leq j \leq n+1 .
$$

The natural transformations $T_{j}$ are given by inclusion for $0 \leq j \leq n$. Gromov's interpolation argument (in [Gro1, 2.3]) and the assumptions in 2.2 above imply that the factorizations $(*)$ exist. (They can be made strict by converting certain maps into fibrations.) Therefore the factorization $(* *)$ exists. Now

$$
\left|\mathcal{Z}_{0}\right| \simeq \bigcup_{1 \leq i \leq N} B_{i}
$$

by 1.3 , and the right-hand side contains $\frac{1}{5} B$. Similarly

$$
\left|\mathcal{Z}_{n+1}\right| \simeq \bigcup_{1 \leq i \leq N} \lambda_{n+1} B_{i}
$$

and the right-hand side is contained in $B$. We conclude that the inclusion

$$
\frac{1}{5} B \hookrightarrow B
$$

has a factorization

$$
\frac{1}{5} B \longrightarrow W \longrightarrow B
$$

where $W$ is homotopy equivalent to a $C W$-space with not more than $p J$ cells. (Never mind the difference between $\left|\mathcal{Z}_{0}\right|$ and $\left|\mathcal{Z}_{0}\right|(n)$ : the inclusion of $\left|\mathcal{Z}_{0}\right|(n)$ in $\left|\mathcal{Z}_{0}\right|$ is $n$-connected, so any map from an $n$-manifold such as $\frac{1}{5} B$ to $\left|\mathcal{Z}_{0}\right|$ can be deformed into $\left|\mathcal{Z}_{0}\right|(n)$.) This shows that the cell content of $B$ is at most $p J$.

Proof of 3.1. Without loss of generality, $\operatorname{dim}(\mathcal{A}) \leq n$, and then $\left|\mathcal{Z}_{0}\right|(n)=\left|\mathcal{Z}_{0}\right|$. Define a new contravariant functor $\mathcal{Y}$ from $\mathcal{A}$ to spaces by

$$
\mathcal{Y}(s)=Y_{i, s} \quad \text { where } i=n-\operatorname{dim}(s)
$$

Induced maps are defined as follows. For simplices $s \subset t$ (proper inclusion) and $i=n-\operatorname{dim}(s)$ and $j=n-\operatorname{dim}(t)$ use the composition

$$
Y_{j, t} \stackrel{\beta_{j, t}}{\longrightarrow} \mathcal{Z}_{j+1}(t) \longrightarrow \mathcal{Z}_{i}(t) \stackrel{\mathcal{Z}_{i}(s \subset t)}{\longrightarrow} \mathcal{Z}_{i}(s) \stackrel{\alpha_{i, s}}{\longrightarrow} Y_{i, s}
$$

where the unlabelled arrow is a specialization of $T_{i-1} \cdots T_{j+1}$, or the identity if $i$ equals $j+1$. (Check that this gives a functor.) There are obvious natural transformations

$$
\mathcal{Z}_{0} \longrightarrow \mathcal{Y} \longrightarrow \mathcal{Z}_{n+1}
$$

with composition equal to $T_{n} T_{n-1} \cdots T_{0}$. Hence we have a factorization

$$
\left|\mathcal{Z}_{0}\right| \longrightarrow|\mathcal{Y}| \longrightarrow\left|\mathcal{Z}_{n+1}\right|
$$

of the map induced by $T_{n} \cdots T_{0}$. To complete the proof, apply the next lemma.

Lemma 3.3. Let $\mathcal{A}$ be a simplicial complex with $J$ simplices, and let $\mathcal{Y}$ be a contravariant functor from $\mathcal{A}$ to spaces. Assume that each $\mathcal{Y}(s)$ is homotopy equivalent to a $C W$-space with at most $p$ cells. Then $|\mathcal{Y}|$ is homotopy equivalent to a $C W$ space with at most $\mathrm{pJ}$ cells.

Proof. Use induction on $J$. For the induction step, let $s$ be a simplex of maximal dimension in $\mathcal{A}$, and let $\mathcal{A}^{\prime}$ be the complement of the interior of $s$ in $\mathcal{A}$. Let $\mathcal{Y}^{\prime}$ be the restriction of $\mathcal{Y}$ to $\mathcal{A}^{\prime}$. Note that $|\mathcal{Y}|$ is the pushout of a diagram

$$
\mathcal{Y}(s) \times s \hookleftarrow \mathcal{Y}(s) \times \partial s \rightarrow\left|\mathcal{Y}^{\prime}\right|
$$




\section{Cell Rank}

Now switch to $\S 1$ of [Abr2]. (I shall use somewhat different notation to be consistent, starting with $V, U, U_{0}$ where Abresch writes $M, X, Y$, respectively.) For open subsets $U_{0} \subset U$ of the manifold $V^{n}$ and $t>0$, Abresch defines

$$
\begin{aligned}
\operatorname{rk}_{j}\left(U, U_{0}\right) & :=\operatorname{rank}\left(H_{j}\left(U_{0} ; F\right) \rightarrow H_{j}(U ; F)\right) \\
\operatorname{rk}_{*}^{t}\left(U, U_{0}\right) & :=\sum_{j \geq 0} \operatorname{rk}_{j}\left(U, U_{0}\right) \cdot t^{j} .
\end{aligned}
$$

Supposing that $B_{i}^{0} \subset B_{i}^{1} \subset \cdots \subset B_{i}^{n+1}$, for $1 \leq i \leq N$, are open subsets of $V$ such that

$$
U_{0} \subset \bigcup_{i=1}^{N} B_{j}^{0} \quad \text { and } \quad U \supset \bigcup_{i=1}^{N} B_{i}^{n+1},
$$

he states the following lemma (which replaces 2.1).

Quotation 4.1. Let $t>0, t^{-1} \in \mathbb{N}$, and suppose that any $B_{i}^{n}$ intersects at most $t^{-1}$ distinct sets $B_{k}^{n}, i \neq k$; then there holds the following inequality:

$$
\begin{aligned}
& \operatorname{rk}_{*}^{t}\left(U, U_{0}\right) \leq \operatorname{rk}_{*}^{t}\left(\bigcup_{i=1}^{N} B_{i}^{n+1}, \bigcup_{i=1}^{N} B_{i}^{0}\right) \\
& \leq(e-1) N \cdot \sup \left\{\operatorname{rk}_{*}^{t}\left(\bigcap_{i \in S} B_{i}^{j+1}, \bigcap_{i \in S} B_{i}^{j}\right) \mid 0 \leq j \leq n, \emptyset \neq S \subset\{1, \ldots, N\}\right\} .
\end{aligned}
$$

Definition 4.2. For a compact $C W$-space $Y$ and $t>0$, define

$$
\sharp^{t}(Y):=\sum_{j \geq 0}(\text { number of } j \text {-cells in } Y) \cdot t^{j} \text {. }
$$

For open subsets $U_{0} \subset U$ in $V$ and $t>0$, let $\operatorname{crk}_{*}^{t}\left(U, U_{0}\right)$ be the minimum of all numbers $q \in \mathbb{N}$ such that there exist maps

$$
U_{0} \stackrel{f}{\rightarrow} Y \stackrel{g}{\rightarrow} U
$$

where $Y$ is a compact $C W$-space with $\sharp^{t}(Y) \leq q$ and $g f$ is homotopic to the inclusion. If there is no such $q$ let $\operatorname{crk}_{*}^{t}\left(U, U_{0}\right)=\infty$.

Lemma 4.3. With $\mathrm{rk}_{*}^{t}$ replaced by $\mathrm{crk}_{*}^{t}$ throughout, the inequality in 4.1 remains correct.

Proof. Let $\mathcal{A}$ be the nerve of the collection of open sets $\left\{B_{i}^{n+1}\right\}$, where $1 \leq i \leq N$. As in 3.2, define contravariant functors $\mathcal{Z}_{j}$ from $\mathcal{A}$ to spaces:

$$
\mathcal{Z}_{j}(s)=\bigcap_{i \text { vertex of } s} B_{i}^{j}
$$

where $0 \leq j \leq n+1$. As in 3.2 , there are natural transformations $\mathcal{Z}_{j} \rightarrow \mathcal{Z}_{j+1}$ for $0 \leq j \leq n$, given by inclusion. Let

$$
p=\sup \left\{\operatorname{crk}_{*}^{t}\left(\bigcap_{i \in S} B_{i}^{j+1}, \bigcap_{i \in S} B_{i}^{j}\right) \mid 0 \leq j \leq n, \emptyset \neq S \subset\{1, \ldots, N\}\right\} .
$$


As in $3.1(* *)$ and the proof of 3.1 , we can construct a factorization

$$
\left|\mathcal{Z}_{0}\right|(n) \longrightarrow W \longrightarrow\left|\mathcal{Z}_{n+1}\right|
$$

where $W=|\mathcal{Y}|$ is the geometric realization of a contravariant functor $\mathcal{Y}$ from the $n$-skeleton $\mathcal{A}^{n}$ to spaces, and $\mathcal{Y}(s)$ is homotopy equivalent to a $C W$-space $X(s)$ such that

$$
\sharp^{t}(X(s)) \leq p
$$

for every face $s \subset \mathcal{A}^{n}$. We now have to show that

$$
\operatorname{crk}_{*}^{t}(|\mathcal{Y}|) \leq(e-1) N p
$$

To this end we show first that $\sharp^{t}\left(\mathcal{A}^{n}\right) \leq(e-1) N$, using the hypotheses in , 4.1 ; this is actually carried out in [Abr2, p.479]. (Beware that our $t$ is Abresch's $t^{-1}$.) Then we finish with a variation on 3.3 :

Lemma 4.4. Let $\mathcal{B}$ be a compact simplicial complex with $\sharp^{t}(\mathcal{B})=J$ and let $\mathcal{Y}$ be a contravariant functor from $\mathcal{B}$ to spaces. Assume that each $\mathcal{Y}(s)$ is homotopy equivalent to a $C W$-space $X(s)$ with $\sharp^{t}(X(s)) \leq p$. Then $|\mathcal{Y}|$ is homotopy equivalent to a $C W$-space $X$ with $\sharp^{t}(X) \leq p J$.

The proof is by induction on the number of simplices in $\mathcal{B}$, like that of 3.3 .

\section{Big SPACES With SMALl HOMOLOGY}

Here is an example showing that Theorem B is stronger than Theorem A. Let $M$ be a square matrix (size $k \times k$ ) with integer entries such that both $M$ and $M-I_{k}$ have determinant \pm 1 ; for instance, $k=2$ and

$$
M=\left(\begin{array}{ll}
1 & 1 \\
1 & 0
\end{array}\right) .
$$

Let $\pi=\mathbb{Z}$, and let the generator of $\pi$ act on $\mathbb{Z}^{k}$ by $M$. Let $\mu$ be the minimum number of generators of

$$
E=\bigoplus_{i=1}^{s} \mathbb{Z}^{k}
$$

as a $\pi$-module. Then $\mu k \geq s$, because $\operatorname{hom}_{\pi}\left(E, \mathbb{Z}^{k}\right)$ contains a free abelian group of rank $s$. Hence $\mu \geq s / k$. Let $X$ be a wedge of $s k$ spheres of dimension $d>1$, and let $f: X \rightarrow X$ be a homotopy equivalence such that $H_{d}(X)$, with the action of $\pi$ determined by $f_{*}$, is isomorphic to $E$ as a $\pi$-module. Finally let $Y$ be the mapping torus of $f$,

$$
Y=X \times[0,1] /(x, 1) \sim(f(x), 0) .
$$

Then $\pi_{1}(Y)=\pi=\mathbb{Z}$, and $H_{*}(Y ; F) \cong H_{*}\left(S^{1} ; F\right)$ for any field $F$. But the number of cells in any $C W$-space dominating $Y$ is $\geq \mu$, which is $\geq s / k$, which is as large as we please.

To obtain closed manifold examples of the same type, just make sure that $Y$ embeds in a high-dimensional euclidean space. Then take a smooth regular neighbourhood and double along the boundary. 


\section{REFERENCES}

[Abr1] U. Abresch, Lower curvature bounds, Toponogov's theorem, and bounded topology, Ann. Sci. École Norm. Sup. (4) 18 (1985), 651-670. MR 87j:53058

[Abr2] Lower curvature bounds, Toponogov's theorem, and bounded topology, II, Ann. Sci. École Norm. Sup. (4) 20 (1987), 475-502. MR 89d:53080

[AlWa] S. Aloff and N. L. Wallach, An infinite family of distinct 7-manifolds admitting positively curved Riemannian structures, Bull. Amer. Math. Soc. 81 (1975), 93-97. MR 51:6851

[BK] A. K. Bousfield and D. M. Kan, Homotopy limits, completions, and localizations, Lecture Notes in Math., vol. 304, Springer-Verlag, New York-Berlin, 1972. MR 51:1825

[Gro1] M.Gromov, Curvature, diameter and Betti numbers, Comment. Math. Helv. 56 (1981), 179-195. MR 82k:53062

[Gro2] _ Almost flat manifolds, J. Diff. Geom. 13 (1978), 231-243. MR 80h:53041

[Groth] A. Grothendieck, Sur quelques points d'algèbre homologique, Tôhoku Math. J. (2) 9 (1957), 119-221. MR 21:1328

[Se] G. Segal, Classifying spaces and spectral sequences, Inst. Hautes Études Sci. Publ. Math. 34 (1968), 105-112. MR 38:718

Department of Mathematics, University of Michigan, Ann Arbor, Michigan 481091003

Current address, Department of Mathematics, University of Notre Dame, Notre Dame, Indiana 46556

E-mail address: msweiss@math.1sa.umich.edu 